\title{
REFLEXIVE AND DIHEDRAL (CO)HOMOLOGY OF A PRE-ADDITIVE CATEGORY
}

\author{
YASIEN GH. GOUDA
}

(Received 18 June 1998)

\begin{abstract}
The group dihedral homology of an algebra over a field with characteristic zero was introduced by Tsygan (1983). The dihedral homology and cohomology of an algebra with involution over commutative ring with identity, associated with the small category, were studied by Krasauskas et al. (1988), Loday (1987), and Lodder (1993). The aim of this work is concerned with dihedral and reflexive (co)homology of small pre-additive category. We also define the free product of involutive algebras associated with this category and study its dihedral homology group. Finally, following Perelygin (1990), we show that a small pre-additive category is Morita equivalence.
\end{abstract}

2000 Mathematics Subject Classification. 55N91, 55P91, 55Q91.

1. Preliminaries. Suppose that $\Delta \mathscr{D}$ is a small category, with objects, the set $\{[0],[1]$, $\ldots,[n], \ldots\}$, and the following family of morphisms $\delta_{n}^{i}:[n] \rightarrow[n-1], 0 \leq i \leq n$, $\sigma_{n}^{j}:[n] \rightarrow[n+1], 0 \leq i \leq n, \tau_{n}:[n] \rightarrow[n], \rho_{n}:[n] \rightarrow[n]$ such that

$$
\begin{aligned}
& \delta_{n+1}^{j} \delta_{n}^{i}=\delta_{n+1}^{i} \delta_{n}^{i-1}, \quad i<j, \\
& \sigma_{n}^{j} \sigma_{n+1}^{i}=\sigma_{n}^{i} \sigma_{n+1}^{j-1}, \quad i \leq j, \\
& \sigma_{n+1}^{j} \delta_{n}^{1}= \begin{cases}\sigma_{n-2}^{i-1} \delta_{n-1}^{j}, & i \leq j, \\
\operatorname{Id}, & i=j, j+1, \\
\sigma_{n-2}^{i} \delta_{n-1}^{i-1}, & i>j,\end{cases} \\
& \tau_{n} \delta_{n}^{i}=\delta_{n}^{i-1} \tau_{n-1}, \quad 0 \leq i \leq n, \quad\left(\tau_{n}\right)^{n+1}=1_{n}, \quad \rho_{n}^{2}=1, \\
& \boldsymbol{\tau}_{n} \sigma_{n}^{j}=\sigma_{n}^{j-1} \tau_{n+1}, \quad 0 \leq j \leq n, \quad \tau_{n} \rho_{n}=\rho_{n} \boldsymbol{T}_{n}^{-1} .
\end{aligned}
$$

DEFINITION 1.1. The category $\Delta \mathscr{D}$ is called a dihedral category. Note that the category generated by only the morphisms $\delta_{n}^{i}$ and $\sigma_{n}^{j}$ is called a simplicial category and is denoted by $\Delta$, the category generated by $\delta_{n}^{i}, \sigma_{n}^{j}$, and $\tau_{n}$ is a cyclic category and is denoted by $\Delta C$ (see [6]), and the category generated by the family of morphisms $\delta_{n}^{i}$, $\sigma_{n}^{j}$, and $\rho_{n}$ is called a reflexive category and is denoted by $\Delta R$.

DEFINITION 1.2 (see [3]). Let $k$ be a commutative ring with identity and involution. An algebra over $k$ associated with the category $\Delta \mathscr{D}(\Delta R)$ is an algebra with identity generated by the morphisms $\delta_{n}^{i}, \sigma_{n}^{j}, \tau_{n}$, and $\rho_{n}\left(\delta_{n}^{i}, \sigma_{n}^{j}, \rho_{n}\right)$. 
DEFINITION 1.3. For an arbitrary category $\mathfrak{C}$, following [3], for case of presentation, a simplicial object in the category $\mathfrak{C}$ is a functor $\mathscr{F}: \Delta^{\mathrm{op}} \rightarrow \mathfrak{C}$ (the category $\Delta^{\mathrm{op}}$ is the inverse of $\Delta$ ).

DEFINITION 1.4. Following [2] (see also [6]), for an arbitrary category $\mathfrak{C}$, the dihedral (reflexive) object in $\mathbb{C}$ is a functor $\mathscr{F}: \Delta D^{\text {op }} \rightarrow \mathfrak{C},\left(\mathscr{F}_{F}: \Delta \mathscr{R}^{\text {op }} \rightarrow \mathfrak{C}\right)$. If we drop the morphism $\rho_{n}$ from the group family of morphisms $\left(\delta_{n}^{i}, \sigma_{n}^{j}, \tau_{n}, \rho_{n}\right)$, we get a cyclic object of an arbitrary category $\mathfrak{C}$ (see [5]). Suppose that $\mathscr{F}([n])=X_{n}, \mathscr{F}\left(\delta_{n}^{i}\right)=d_{n}^{i}$, $\mathscr{F}\left(\sigma_{n}^{j}\right)=\mathfrak{s}_{n}^{j}, \mathscr{F}\left(\tau_{n}\right)=t_{n}, \mathscr{F}\left(\rho_{n}\right)=\boldsymbol{r}_{n}$. We write the dihedral (reflexive) object by the family $\left(X_{n}, d_{n}^{i}, \mathfrak{s}_{n}^{j}, t_{n}, r_{n}\right)\left(X_{n}, d_{n}^{i}, \mathfrak{s}_{n}^{j}, r_{n}\right)$. We can easily check that the morphisms $d_{n}^{i}, \mathfrak{s}_{n}^{j}, t_{n}$, and $r_{n}$ satisfy relations (1.1).

DEFINITION 1.5. Let $k$ be a commutative ring with identity and involution and let $\mathfrak{C}$ be a category of $k$-modules. The dihedral $k$-module in $\mathfrak{C}$ is defined to be the dihedral objects $\left(X_{n}, d_{n}^{i}, \mathfrak{s}_{n}^{j}, t_{n}, \boldsymbol{r}_{n}\right)$ in the category $\mathfrak{C}$.

2. The reflexive and dihedral homology of pre-additive category. In this section, we define the dihedral $k$-module associated with a pre-additive category and study its (co)homology.

DEFINITION 2.1. Let $k$ be a commutative ring with identity and involution. Following [2], the $k$-category $A$ with an involution is defined to be a small pre-additive category with objects the $k$-modules of set morphisms $A(i, j)$, where, $i, j$ are in $A$, and the bilinear maps $A(i, j) x A(j, k) \rightarrow A(i, k)$, as morphisms. Suppose that, for all objects $i, j \in A$, there exists a $k$-linear map $*: A(i, j) \rightarrow A(j, i)$, such that $*^{2}: A(i, j) \rightarrow A(i, j)$. Define the family $M=\left\{M_{n}\right\}_{n \geq 0}$ of $k$-modules and $k$-morphisms as follows:

$$
M_{0}=\underset{i_{0} \in|A|}{\oplus} A\left(i_{0}, i_{0}\right), \ldots, M_{n}=\underset{i_{0}, i_{1}, \ldots, i_{n} \in|A|}{\oplus} A\left(i_{0}, i_{0}\right) \underset{k}{\otimes} A\left(i_{1}, i_{2}\right) \underset{k}{\otimes} \cdots \underset{k}{\otimes} A\left(i_{n}, i_{0}\right) .
$$

On the family $M=\left(M_{n}\right)$, define the morphisms $d_{n^{\prime}}^{i} \mathbf{s}_{n^{\prime}}^{j} t_{n^{\prime}} r_{n}$ as follows:

$$
d_{n}^{i}: M_{n} \longrightarrow M_{n-1}, \quad \mathfrak{s}_{n}^{j}: M_{n} \longrightarrow M_{n-1}, \quad r_{n}, t_{n}: M_{n} \longrightarrow M_{n},
$$

such that

$$
\begin{aligned}
& d_{n}^{i}\left(a_{0} \otimes \cdots \otimes a_{n}\right)=d_{n}^{i}\left(a_{0} \otimes \cdots \otimes a_{n}\right)+(-1)^{n} a_{n} a_{0} \otimes \cdots \otimes a_{n-1}, \\
& d_{n}^{i}\left(a_{0} \otimes \cdots \otimes a_{n}\right)=\sum_{k=0}^{n-1}(-1)^{k} a_{0} \otimes \cdots \otimes a_{k} a_{k+1} \otimes \cdots \otimes a_{n}, \\
& \mathfrak{s}_{n}^{j}\left(a_{0} \otimes \cdots \otimes a_{n}\right)=a_{0} \otimes a_{1} \otimes \cdots \otimes a_{i} \otimes 1 \otimes a_{i+1} \otimes \cdots \otimes a_{n}, \\
& t_{n}\left(a_{0} \otimes \cdots \otimes a_{n}\right)=(-1)^{n}\left(a_{n} \otimes a_{0} \otimes \cdots \otimes a_{n-1}\right), \\
& r_{n}\left(a_{0} \otimes \cdots \otimes a_{n}\right)=\alpha(-1)^{n(n+1) / 2} a_{0}^{*} \otimes a_{n}^{*} \otimes \cdots \otimes a_{1}^{*}, \quad \alpha= \pm 1,
\end{aligned}
$$

where $a_{i}^{*}$ 's are the image of the elements $a_{i}(0 \leq i \leq n)$ under the involution $*$. Clearly, the module $M=\left\{M_{n}\right\}$ under the last morphisms is a dihedral $k$-module. Now, 
we define the dihedral homology group. Suppose that $M=\left(M_{n}\right)=C^{p, q, r}, p, q, r>0$, and consider the complex $\left(C^{p, q, r}, \delta^{i}\right), i=1,2$ (see [3]), where

$$
\delta^{1}: C^{p, q, r} \longrightarrow C^{p-1, q, r}, \quad \delta^{2}: C^{p, q, r} \longrightarrow C^{p, q-1, r},
$$

are defined by

$$
\begin{aligned}
& \delta^{1}= \begin{cases}1-T_{n}, & n=1(\bmod 2), \\
N_{n}=1+T_{n}+\cdots+T_{n}^{n}, & n=0(\bmod 2),\end{cases} \\
& \delta^{2}= \begin{cases}b_{n+1}=(-1)^{i} d_{n+1}^{i}, & n=0(\bmod 2), \\
-b=(-1)^{i} d^{i}, & n=1(\bmod 2) .\end{cases}
\end{aligned}
$$

Clearly, by definition, $\delta^{i} \cdot \delta^{i}=0, i=1,2$. The complex $\left(C^{p, q, r}, \delta^{i}\right)$ can be illustrated by Tsygan's bicomplex $\mathfrak{C}(M)$ (see [5]):

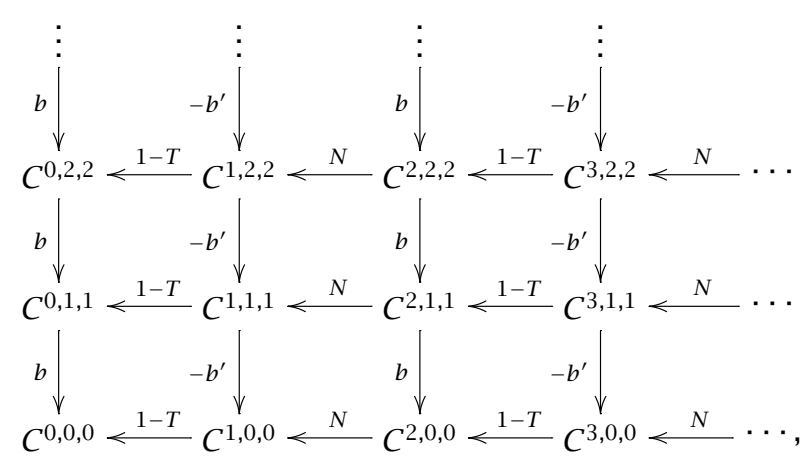

where the morphisms $b,-b^{\prime}: C^{p, q, r} \rightarrow C^{p, q-1, r}$ are given by $b=\sum_{i=0}^{n}(-1)^{i} d_{n}^{i},-b^{\prime}=$ $\sum_{i=0}^{n-1}(-1)^{i} d_{n}^{i}, T=(-1)^{n} t_{n}, N=1+T_{n}+\cdots+T_{n}^{n}$. Following [5], the homology of the bicomplex (2.6) gives the cyclic homology group: $\mathscr{H}_{n}(M)=\mathscr{H}_{n}(\mathfrak{C}(M))$. Following [3], if we act by the group $\mathbb{Z} / 2$ on the bicomplex (2.6): on the column $2 \ell(\ell>0)$ by means of the automorphism

$$
(-1)^{n(n+1) / 2+\ell} \boldsymbol{r}_{n}=(-1)^{\ell} R_{n}, \quad \text { where } R_{n}\left(a_{0} \otimes \cdots \otimes a_{n}\right)=(-1)^{n(n+1) / 2} \boldsymbol{r}_{n},
$$

and on the column $2 \ell+1$ by means of the automorphism $(-1)^{n(n-1) / 2+\ell+1} \boldsymbol{r}_{n}=$ $(-1)^{\ell} R_{n} T_{n}$, we get the tricomplex $\left(C^{p, q, r}, \delta^{i}\right), i=1,2,3$. The differentials $\delta^{1}, \delta^{2}$ are defined in (2.5) and $\delta^{3}: C^{p, q, r+1} \rightarrow C^{p, q, r}$ is defined by

$$
\delta^{3}= \begin{cases}(-1)^{n}\left(1+(-1)^{\ell} R_{n}\right), & n=0(\bmod 4), \\ (-1)^{n+1}\left(1+(-1)^{\ell+1} R_{n} T_{n}\right), & n=1(\bmod 4), \\ (-1)^{n}\left(1+(-1)^{\ell+1} R_{n}\right), & n=2(\bmod 4), \\ (-1)^{n+1}\left(1+(-1)^{\ell+1} R_{n} T_{n}\right), & n=3(\bmod 4), R_{n}=(-1)^{n} r_{n} .\end{cases}
$$

Following [4], the dihedral homology of the module $M(\alpha \mathscr{H} D(M))$ is the homology of the complex $\left(C^{p, q, r}, \delta^{i}\right), i=1,2,3, \alpha= \pm 1$. 
DEFINITION 2.2. The dihedral homology of a $k$-category $A$ with involution is the dihedral homology of the associated dihedral $k$-module $M=\left\{M_{n}\right\}: \alpha \mathscr{H} \mathscr{D}_{n}(A)=\alpha \mathscr{H} \mathscr{D}_{n}(M)$, $\alpha= \pm 1$.

DEFINITION 2.3. The reflexive homology of a $k$-category $A$ with an involution is the reflexive homology of $k$-module $\left\{M_{\text {refl }}\right\}$ :

$$
\alpha \mathscr{H}_{n}(A)=\alpha \mathscr{H}_{n}\left(M_{\text {refl }}\right), \quad \alpha \pm 1,
$$

where $M_{\text {refl }}$ is the reflexive $k$-module $M=\left\{M_{n}\right\}$. Similarly, if we take the cyclic $k$ module $M_{\text {cycl }}$, we obtain the cyclic homology of the $k$-category $A$ (see [6]): $\mathscr{H} C_{n}(A)=$ $\mathscr{H} \mathscr{R}_{n}\left(M_{\text {cycl }}\right)$. Following [3, 4], the dihedral (reflexive) homology of the dihedral (reflexive) module $M$ can be considered as derived functor

$$
\alpha \mathscr{H} \mathscr{D}_{n}(M)=\operatorname{Tor}_{n}^{k[\Delta D]^{\mathrm{op}}}\left(k^{\mathscr{D}} \cdot M \cdot\right)\left(\alpha \mathscr{H} \mathscr{R}_{n}(M)=\operatorname{Tor}_{n}^{k[\Delta R]^{\mathrm{op}}}\left(k^{\mathscr{R}} \cdot M \cdot\right)\right),
$$

where $k^{\mathscr{D}}\left(k^{\mathscr{R}}\right)$ is a trivial dihedral (reflexive) $k$-module, $k[\Delta D]^{\mathrm{op}}\left(k[\Delta R]^{\mathrm{op}}\right)$ is the algebra associated with the dihedral (reflexive) category.

Note that (see [3]) the dihedral (reflexive) homology is considered as the hyperhomology of the group $\mathbb{Z} / 2$ with coefficients in Tsygan bicomplex (simplicial (Hochschild) complex). The relations between the cyclic and the dihedral homology and also the reflexive and the dihedral homology of pre-additive category are given by the following assertions.

THEOREM 2.4. Let $k$ be a commutative ring and let $A$ be a $k$-category with an involution. Then there exist the following exact sequences

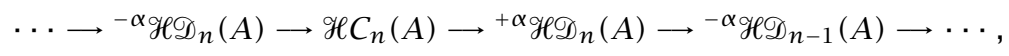

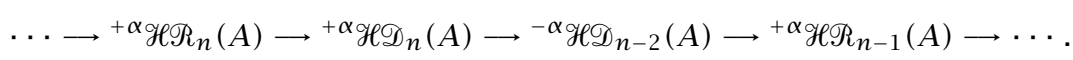

Proof. The proof follows from [3].

COROLLARY 2.5. Let $1 / 2 \in k$. Then there exists the natural isomorphism

$$
\mathscr{H C}_{n}(A) \cong-\alpha \mathscr{H} \mathscr{D}_{n}(A) \oplus^{+\alpha} \mathscr{H}_{n}(A) .
$$

Note that we can define the reflexive cohomology and the dihedral cohomology of a pre-additive category in the same manner.

3. The dihedral homology of free product algebras. In this section, we study the product of the algebras associated with a pre-additive $k$-category, where $k$ has characteristic zero. Let $A, B$, and $C$ be arbitrary involutive algebras. The free product of the algebras $A$ and $B$ with respect to algebra $C$ is denoted by $A \underset{C}{*} B$. Following [1], the algebra $A \underset{C}{*} B$ is $C$-bimodule. For the algebras $A, B$, and $C$,

$$
\operatorname{Tor}_{i}^{C}(A, A)=\operatorname{Tor}_{i}^{C}(A, B)=\operatorname{Tor}_{i}^{C}(B, A)=\operatorname{Tor}_{i}^{C}(B, B)=0
$$


Consider also the following diagram of algebras and homomorphisms between them:

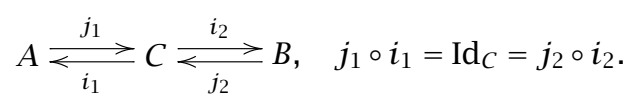

Following [3], let $R^{A}$ and $R^{B}$ be the free involutive resolution of the algebras $A$ and $B$ over the homomorphisms $i_{1}$ and $i_{2}$, respectively. Consequently, we get the following diagram:

$$
R^{A} \stackrel{i_{1}^{A}}{\longleftarrow} \stackrel{i_{2}^{B}}{\longrightarrow} R^{B}
$$

Consider the diagrams

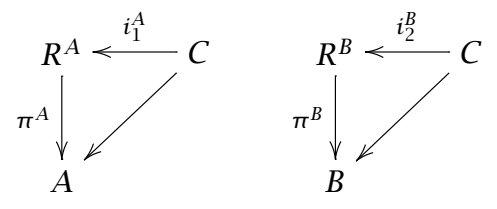

Clearly, they are commutative. If we define the homomorphisms $j_{1}^{A}$ and $j_{2}^{B}$ as follows:

$$
R^{A} \stackrel{j_{1}^{A}}{\longrightarrow} R^{B}, \quad R^{B} \stackrel{j_{2}^{B}}{\longrightarrow} C, \quad \text { where } j_{1}^{A}=j_{1} \circ \pi^{A}, j_{2}^{B}=j_{2} \circ \pi^{A},
$$

we get the diagram

$$
R^{A} \underset{i_{1}^{A}}{\stackrel{j_{1}^{A}}{\ll}} C \underset{j_{2}^{B}}{\stackrel{i_{2}^{B}}{\gtrless}} R^{B},
$$

where $j_{1}^{A} \circ i_{1}^{A}=\operatorname{Id}_{C}, j_{2}^{B} \circ i_{2}^{B}=\operatorname{Id}_{C}$, since $j_{1}^{A} \circ i_{1}^{A}=\left(j_{1} \circ \pi^{A}\right) \circ i_{1}^{A}=j_{1} \circ i_{1}=\operatorname{Id}_{c}, j_{2}^{B} \circ i_{2}^{B}=$ $\left(j_{2} \circ \pi^{B}\right) \circ i_{2}^{B}=j_{2} \circ i_{2}=\operatorname{Id}_{c}$. Suppose that $\bar{R}^{A}=\operatorname{ker} j_{1}^{A}, \bar{R}^{B}=\operatorname{ker} j_{2}^{B}$. Then $R^{A} \underset{c}{*} R^{B}$ is a $C$-module given by

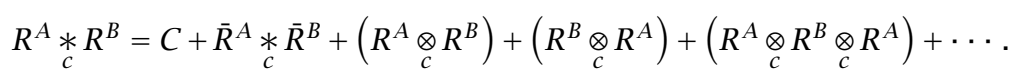

We define an involution on $R^{A} \underset{c}{*} R^{B}$ as follows:

$$
\begin{aligned}
& \left(p_{0} q_{0} p_{1} q_{1} \cdots p_{n} q_{n} q_{n+1}\right)^{*} \\
& \quad=p_{n+1}^{*} q_{n}^{*} p_{n}^{*} \cdots q_{0}^{*} p_{0}^{*}, \quad p_{i} \in R^{A}, 0 \leq i \leq n+1, q_{i} \in R^{B}, 0 \leq i \leq n .
\end{aligned}
$$

REMARKS. (i) The differential on $R_{c}^{A} \underset{c}{*} R^{B}$ is defined by Leibniz formula for differential graded algebras [5].

(ii) The chain complex $\bar{R}^{A}$ is a free $C$-biomodule resolution of the $C$-biomodule $\bar{A}$.

(iii) $\bar{A}+C=A=H \cdot\left(R^{A}\right)=H \cdot\left(\bar{R}^{A} \oplus C\right)=H \cdot\left(\bar{R}^{A}\right)+C$, that is, $H \cdot\left(\bar{R}^{A}\right)=\bar{A}$, where $H$ is a hyperhomology of $\bar{R}^{A}$. From (3.1), (i), (ii), and (iii), we have

$$
\operatorname{Tor}_{i}^{c}(\bar{A}, \bar{A})=\operatorname{Tor}_{i}^{c}(\bar{A}, \bar{B})=\operatorname{Tor}_{i}^{c}(\bar{B}, \bar{A})=\operatorname{Tor}_{i}^{c}(\bar{B}, \bar{B})=0
$$


From (3.7) and (3.9), we have $A \underset{c}{*} B=H \cdot\left(\bar{R}_{c}^{A} \underset{c}{\otimes} \bar{R}^{B}\right)$ (see [1]). Consider the following diagram:

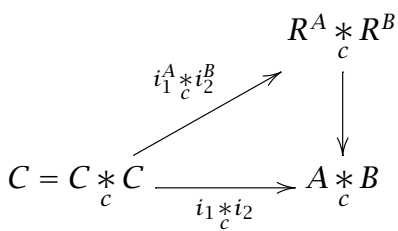

LEMMA 3.1. The diagram (3.10) is commutative.

PROoF. The proof follows from the fact that the differential graded algebra $R_{c}^{A} * R^{B}$ is an involutive resolution of the algebra $A \underset{c}{*} B$ over the inclusion $i_{1} \underset{c}{*} i_{2}$.

Consider the complex $T^{(n)}\left(C ; \bar{R}^{A} \underset{c}{\otimes} \bar{R}^{B}\right)=C \underset{c \otimes c^{\text {op }}}{\otimes}\left(\left(\bar{R}^{A} \underset{c}{\otimes} \bar{R}^{B}\right) \otimes \cdots \otimes\left(\bar{R}^{A} \otimes \bar{R}^{B}\right)\right)$. We act on the complex by means of an automorphism ${ }^{\epsilon} \gamma_{n}$ as follows:

$$
{ }^{\epsilon} \gamma_{n}\left(\left(p_{0} \otimes q_{0}\right) \otimes \cdots \otimes\left(p_{n} \otimes q_{n}\right)\right)=(-1)^{\ell+v} \epsilon\left(p_{0}^{*} \otimes q_{n}^{*}\right) \otimes\left(p_{n}^{*} \otimes q_{n-1}^{*}\right) \otimes \cdots \otimes\left(p_{1}^{*} \otimes q_{0}^{*}\right)
$$

where $p_{i} \in \bar{R}^{A}, q_{i} \in \bar{R}^{B}, 0 \leq i \leq n, \ell=\operatorname{deg} p_{0} \sum_{i=1}^{n} \operatorname{deg} p_{i}+\sum_{j=1}^{n} \operatorname{deg} q_{i}, v=\alpha(\alpha-1) / 2$, $\alpha=\sum_{i=1}^{n} \operatorname{deg} p_{i}+\operatorname{deg} q_{i}, \alpha= \pm 1$. Consider the chain complex homomorphism

$$
\mu: \frac{T^{(n)}\left(C ; R_{c}^{A} \underset{*}{*} R^{B}\right)}{\operatorname{Im}\left(1-t_{n}\right)+\operatorname{Im}\left(1-{ }^{\epsilon} \gamma_{n}\right)} \rightarrow \frac{\left(R^{A} * R^{B}\right)}{C+R^{A}+R^{B}+\left[R_{c}^{A} \underset{c}{*} R^{B}, R^{A} \underset{c}{*} R^{B}\right]+\operatorname{Im}\left(1-{ }^{\epsilon} \gamma_{n}\right)},
$$

such that

$$
\begin{aligned}
& \mu\left(\left(\left(p_{0} \otimes q_{0}\right) \otimes \cdots \otimes\left(p_{n} \otimes q_{n}\right)\right) \bmod \left(\operatorname{Im}\left(1-t_{n}\right)+\operatorname{Im}\left(1-{ }^{\epsilon} \gamma_{n}\right)\right)\right) \\
& \quad=\left(p_{0} q_{0} \cdots p_{n} q_{n}\right) \bmod \left(C+\bar{R}^{A}+\bar{R}^{B}+\left[R^{A} \underset{c}{*} R^{B}, R^{A} \underset{c}{*} R^{B}\right]+\operatorname{Im}\left(1-{ }^{\epsilon} \mathfrak{r}\right)\right) .
\end{aligned}
$$

In the following lemma, we explain the existence of the homomorphism $\mu$ and prove that it is an isomorphism.

LEMMA 3.2. A chain complex homomorphism $\mu$ is an isomorphism.

Proof. Clearly, in $R_{c}^{A} * R^{B}$, there exists a subcomplex $C+\bar{R}^{A}+\bar{R}^{B}$ (but in $T^{(n)}(C$; $\bar{R}^{A} \otimes \bar{R}^{B}$ ) there is not), and we can factorize $R^{A} * R^{B}$ by this subcomplex. The elements in $\bar{R}^{A}{ }_{c} \bar{R}^{B}$ can be compared by modulo with the commutant of the algebra $R_{c}^{A} * R^{B}$ with elements in $\bar{R}^{A} \underset{c}{\otimes} \bar{R}^{B}$ since $\mathscr{L} \otimes a+(-1)^{m}\left(a \otimes \mathscr{L}-(-1)^{(\operatorname{deg} a) \cdot(\operatorname{deg} \mathscr{L})} \mathscr{L} \otimes a\right)=(-1)^{m} a \otimes \mathscr{L}$, where

$$
m= \begin{cases}0, & \text { if }(\operatorname{deg} a) \cdot(\operatorname{deg} \mathscr{L}) \text { is even, } \\ 1, & \text { if }(\operatorname{deg} a) \cdot(\operatorname{deg} \mathscr{L}) \text { is odd, } a \in \bar{R}^{A}, \mathscr{L} \in \bar{R}^{B} .\end{cases}
$$


Note that the elements in $\bar{R}^{A} \underset{c}{\otimes} \bar{R}^{B} \underset{c}{\otimes} \bar{R}^{A}$ or in $\bar{R}_{c}^{B} \underset{c}{\otimes} \bar{R}_{c}^{A} \underset{c}{\otimes} \bar{R}^{B}$ can also be compared by modulo with the commutant of the algebra $R_{c}^{A} \underset{c}{*} R^{B}$ with elements in $\bar{R}_{c}^{A} \underset{c}{\otimes} \bar{R}^{B}$, at the same time;

$p q p^{\prime}-\left(p q p^{\prime}-(-1)^{\operatorname{deg}(p \cdot q) \cdot \operatorname{deg} p^{\prime}} p^{\prime} p q\right)=(-1)^{\operatorname{deg}(p q) \cdot \operatorname{deg} p^{\prime}}\left(p^{\prime} p\right) q, \quad p, p^{\prime} \in \bar{R}^{A}, q \in \bar{R}^{B}$.

In the complex $T^{(n)}\left(C ; \bar{R}^{A} \underset{c}{\otimes} \bar{R}^{B}\right) / \operatorname{Im}\left(1-t_{n}\right)+\operatorname{Im}\left(1-{ }^{\epsilon} \gamma_{n}\right)$, we have the following:

$$
\left(p_{0} \otimes q_{0}\right) \otimes \cdots \otimes\left(p_{n} \otimes q_{n}\right)=(-1)^{S}\left(p_{n} \otimes q_{n}\right) \otimes\left(p_{0} \otimes q_{0}\right) \otimes \cdots \otimes\left(p_{n-1} \otimes q_{n-1}\right),
$$

where $s=\operatorname{deg}\left(p_{n} \otimes q_{n}\right) \sum_{i=1}^{n-1} \operatorname{deg}\left(p_{i} \otimes q_{i}\right)$ and $\left(p_{0} \otimes q_{0}\right) \otimes \cdots \otimes\left(p_{n} \otimes q_{n}\right)=(-1)^{\ell+v} \epsilon$ $\left(p_{0}^{*} \otimes q_{n}^{*}\right) \otimes\left(p_{n}^{*} \otimes q_{n-1}^{*}\right) \otimes \cdots \otimes\left(p_{1}^{*} \otimes q_{0}^{*}\right) ; \ell=\operatorname{deg} p_{0} \cdot \sum_{i=1}^{n} \operatorname{deg} p_{i}+\sum_{j=1}^{n} \operatorname{deg} q_{j}$, $v=\alpha(\alpha-1) / 2, \alpha=\sum_{i=1}^{n} \operatorname{deg} p_{i}+\operatorname{deg} q_{i}$. Clearly, the same relation holds in the complex: $\left(R_{c}^{A} \underset{c}{*} R^{B}\right) / C+\bar{R}^{A}+\bar{R}^{B}+\left[R_{c}^{A} \underset{c}{*} R^{B}, R_{c}^{A} \underset{c}{*} R^{B}\right]+\operatorname{Im}\left(1-\epsilon_{\boldsymbol{r}}\right)$ since

$$
\left(p_{0} q_{0} p_{1} q_{1} \cdots p_{n-1} q_{n-1}\right)\left(p_{n} q_{n}\right)=(-1)^{S^{\prime}}\left(p_{n} q_{n}\right)\left(p_{0} q_{0} p_{1} q_{1} \cdots p_{n-1} q_{n-1}\right),
$$

where $s^{\prime}=\operatorname{deg}\left(p_{n} q_{n}\right) \sum_{i=1}^{n-1} \operatorname{deg}\left(p_{i} q_{i}\right)$, and since $\operatorname{deg}\left(p_{n} q_{n}\right)=\operatorname{deg}\left(p_{n} \otimes q_{n}\right), s=s^{\prime}$ and

$$
\begin{aligned}
p_{0} q_{0} p_{1} q_{1} \cdots p_{n} q_{n} & =(-1)^{v} q_{n}^{*} p_{n}^{*} \cdots q_{1}^{*} p_{1}^{*} q_{0}^{*} p_{0}^{*} \\
& =(-1)^{v+\ell}\left(p_{0}^{*} q_{n}^{*}\right)\left(p_{n}^{*} q_{n-1}^{*}\right) \cdots\left(p_{2}^{*} q_{1}^{*}\right)\left(p_{1}^{*} q_{0}^{*}\right) .
\end{aligned}
$$

This gives the required isomorphism. It is easily seen that the differentials in the complexes $\otimes_{n=0}^{\infty} T^{(n)}\left(C ; \bar{R}^{A}{\underset{c}{\otimes}}^{B}\right) / \operatorname{Im}\left(1-t_{n}\right)+\operatorname{Im}\left(1-{ }^{\epsilon} \gamma_{n}\right)$ and $\left(R^{A} * R^{B}\right) / C+\bar{R}^{A}+\bar{R}^{B}+$ $\left[R^{A}{ }_{c} R^{B}, R_{c}^{A}{ }_{c}^{*} R^{B}\right]+\operatorname{Im}\left(1-\epsilon_{\mathfrak{r}}\right)$ coincide. Using the condition $\operatorname{Tor}_{i}^{c}(\bar{A}, \bar{B})=0, i>0$, we find that $\bar{R}^{A}{\underset{c}{C}}^{B}$ is a free $C$-module resolution of the algebra $\bar{R} \underset{c}{\otimes} \bar{R}$. Then by considering the isomorphism $\mu$, we get

$$
\mathscr{H} \cdot\left(\frac{R_{c}^{A} \underset{c}{*} R^{B}}{C+\bar{R}^{A}+\bar{R}^{B}+\left[R_{c}^{A} \underset{c}{*} R^{B}, R^{A} \underset{c}{*} R^{B}\right]+\operatorname{Im}(1-\epsilon \gamma)}\right)=\frac{\Omega(C, \bar{A} \otimes \bar{B})}{\operatorname{Im}\left(1-\underset{n=0}{\infty} \epsilon \tilde{\gamma}_{n}\right)},
$$

where ${ }^{\epsilon} \tilde{\gamma}_{n}$ is an automorphism on the graded $K$-module $H \cdot\left(\mathbb{Z} /(n+1) ; T^{(n)}\left(C ; \bar{R}^{A} \underset{c}{\otimes}\right.\right.$ $\left.\bar{R}^{B}\right)$ ) is induced by the automorphism ${ }^{\epsilon} \gamma_{n}$ on the complex $T^{(n)}\left(C ; \bar{R}^{A} \underset{c}{\otimes} \bar{R}^{B}\right)$. From the isomorphism $\mu$, we get the following isomorphism:

$$
\begin{aligned}
& \epsilon \mathscr{H} \mathscr{D}_{i}\left(C \stackrel{i_{1}}{\longrightarrow} A\right) \oplus \epsilon \mathscr{H}_{i}\left(C \stackrel{i_{2}}{\longrightarrow} B\right) \oplus\left\{\frac{\stackrel{\Omega}{\sim}_{i}(C, \bar{A} \otimes \bar{B})}{\operatorname{Im}\left(1-\underset{n=0}{\oplus} \epsilon \tilde{\gamma}_{n}\right)}\right\} \\
& =\mathscr{H} \cdot\left(\frac{R_{c}^{A} \underset{c}{*} R^{B}}{C+\bar{R}^{A}+\bar{R}^{B}+\left[R_{c}^{A} \underset{c}{*} R^{B}, R^{A} \underset{c}{*} R^{B}\right]+\operatorname{Im}\left(1-\epsilon_{\mathfrak{r}}\right)}\right) \\
& \oplus \mathscr{H}^{\mathcal{H} \mathscr{D}_{i}}\left(C \stackrel{i_{1}}{\longrightarrow} A\right) \oplus \oplus^{\epsilon} \mathscr{H}_{\mathscr{D}_{i}}\left(C \stackrel{i_{2}}{\longrightarrow} B\right) \text {. }
\end{aligned}
$$

LEMMA 3.3. The right-hand side of relation (3.20) is isomorphic to the group $\epsilon \mathscr{H} \mathscr{D}_{i}\left(C \stackrel{i_{1} * i_{2}}{\longrightarrow} A \underset{c}{*} B\right)$. 
Proof. Since

$$
\begin{aligned}
& \frac{R_{c}^{A} \underset{c}{*} R^{B}}{\left(C+\left[R^{A} \underset{c}{*} R^{B}, R^{A} \underset{c}{*} R^{B}\right]+\operatorname{Im}\left(1-\epsilon_{\mathfrak{r}}\right)\right)} \\
& =\frac{\left(R_{c}^{A} \underset{c}{*}\right)}{C+\left[R^{A} \underset{c}{*} R^{B}, R_{c}^{A} \underset{c}{*}\right]+\operatorname{Im}\left(1-\epsilon_{\mathfrak{r}}\right)+\bar{R}^{A}+\bar{R}^{B}} \\
& +\left\{\frac{\bar{R}^{A}}{\left[\bar{R}^{A}, \bar{R}^{A}\right]+\operatorname{Im}\left(1-{ }^{\epsilon} \boldsymbol{r}_{R} A\right)}\right\}+\left\{\frac{\bar{R}^{B}}{\left[\bar{R}^{B}, \bar{R}^{B}\right]+\operatorname{Im}\left(1-\epsilon_{\left.\mathfrak{r}_{R} B\right)}\right.}\right\} \\
& =\left\{\frac{R^{A} * R^{B}}{C+\left[R_{c}^{A} \underset{c}{*} R^{B}, R^{A} \underset{c}{*} R^{B}\right]+\operatorname{Im}\left(1-\epsilon_{\boldsymbol{r}}\right)+\bar{R}^{A}+\bar{R}^{B}}\right\} \\
& +\left\{\frac{R^{A}}{C+\left[R^{A}, R^{A}\right]+\operatorname{Im}\left(1-{ }^{\epsilon} \boldsymbol{r}_{R} A\right)}\right\}+\left\{\frac{R^{B}}{C+\left[R^{B}, R^{B}\right]+\operatorname{Im}\left(1-{ }^{\epsilon} \mathfrak{r}_{R} B\right)}\right\},
\end{aligned}
$$

we have

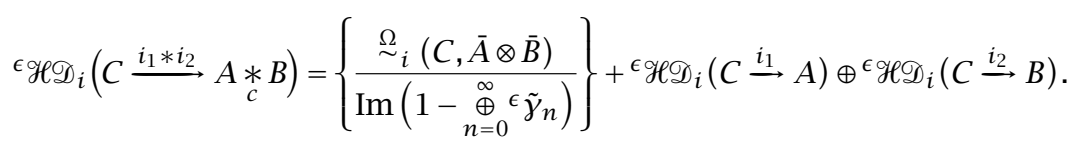

LEMMA 3.4. The following isomorphism holds:

$$
\epsilon \mathscr{H}_{i}(A \underset{c}{*} B) \oplus \epsilon \mathscr{H}_{i}(C)=\epsilon \mathscr{H}_{i}(A) \oplus \epsilon \mathscr{H}_{i}(B) \oplus \frac{\stackrel{\Omega}{\sim}_{i}(C, \bar{A} \oplus \bar{B})}{\operatorname{Im}\left(1-\underset{n=0}{\infty} \epsilon \tilde{\gamma}_{n}\right)} .
$$

Proof. This follows from the fact that

$$
\begin{aligned}
& \epsilon \mathscr{H}_{i}(A)=\epsilon \mathscr{H D}_{i}\left(C \stackrel{i_{1}}{\longrightarrow} A\right) \oplus \epsilon \mathscr{H} \mathscr{D}(C), \\
& \epsilon \mathscr{H}_{D_{i}}(B)=\epsilon \mathscr{H}_{D_{i}}\left(C \stackrel{i_{2}}{\longrightarrow} B\right) \oplus \epsilon \mathscr{H} \mathscr{D}(C), \\
& \epsilon \mathscr{H}_{i}(A \underset{c}{*} B)=\operatorname{EH}_{i}\left(C \stackrel{i_{1} *_{c}^{i_{2}}}{\longrightarrow} A \underset{c}{*} B\right) \oplus \epsilon \mathscr{H}(C) .
\end{aligned}
$$

Note that the last three relations are obtained from the long exact sequence of relative dihedral homology of algebras [5]. Following [3] (also, see [4]), the automorphisms $t_{n}$ and ${ }^{\epsilon} \gamma_{n}$ give the representation of the dihedral group $\mathscr{D}_{n+1}$ on the complex $T^{(n)}\left(C ; \bar{R}^{A} \otimes \bar{R}^{B}\right)$, where $\left(t_{n}\right)^{n+1}=\left({ }^{\epsilon} \gamma_{n}\right)^{2}=\mathrm{Id},{ }^{\epsilon} \gamma_{n} t_{n}^{n}=t_{n}^{-1 \epsilon} \gamma_{n}$. Then if $\operatorname{char}(k)=0$, we get the following isomorphism:

$$
\frac{\Omega_{i}(C, \bar{A} \otimes \bar{B})}{\operatorname{Im}\left(1-\underset{n=0}{\infty} \epsilon \tilde{\gamma}_{n}\right)} \cong \underset{n=0}{\oplus} \mathbb{\leftrightarrow} \cdot\left(\mathscr{D}_{n+1} ; T^{(n)}\left(C ; \bar{R}^{A} \underset{c}{\otimes} \bar{R}^{B}\right)\right) .
$$

From Lemma 3.3 and relation (3.20), we get

$$
\begin{aligned}
& \epsilon \mathscr{H}_{i}(A \underset{c}{* B} B) \oplus \epsilon \mathscr{H}_{i}(C) \\
& =\epsilon \mathscr{H}_{i}(A) \oplus \epsilon \mathscr{H}_{i}(B) \oplus\left(\underset{n=0}{\oplus} \mathbb{\boxplus} \cdot\left(\mathscr{D}_{n+1} ; T^{(n)}\left(C ; \bar{R}^{A} \underset{c}{\otimes} \bar{R}^{B}\right)\right)\right) .
\end{aligned}
$$

So, we have proved the following theorem. 
THEOREM 3.5. Consider the following diagram of involutive algebras associated with a pre-additive $\mathrm{K}$-category:

$$
A \underset{i_{1}}{\stackrel{j_{1}}{\gtrless}} C \underset{j_{2}}{\stackrel{i_{2}}{\gtrless}} B, \quad \text { where } j_{1} \circ i_{1}=\operatorname{Id}_{C}=j_{2} \circ i_{2} \text {. }
$$

If

$$
\operatorname{Tor}_{i}^{C}(A, A)=\operatorname{Tor}_{i}^{C}(A, B)=\operatorname{Tor}_{i}^{C}(B, A)=\operatorname{Tor}_{i}^{C}(B, B)=0, \quad i>0,
$$

then we have

$$
\begin{aligned}
\epsilon \mathscr{H}_{i}(A & \underset{c}{*} B) \oplus \operatorname{HHD}_{i}(C) \\
& ={ }^{\epsilon} \mathscr{H}_{i}(A) \oplus \epsilon \mathscr{H}_{i}(B) \oplus\left(\underset{n=0}{\oplus} \mathbb{Q} \cdot\left(\mathscr{D}_{n+1} ; T^{(n)}\left(C ; \bar{R}^{A} \underset{c}{\otimes} \bar{R}^{B}\right)\right)\right) .
\end{aligned}
$$

Let $A$ be a $k$-category with an involution, and let $\operatorname{Mod} A$ be the category of right $A$-modules and $P(A)$ be full subcategory in $\operatorname{Mod} A$, consisting of the finite projective modules. Consider the category $M_{\mathrm{rt}}(k)$ with objects, the $k$-categories with involution and morphisms $f: A \rightarrow B$ are $k$-factors $F: \operatorname{Mod} A \rightarrow \operatorname{Mod} B$, such that for every $X \in$ $P(A), f(X) \in P(B), f$ commutes with an involution. We call these morphisms, Moritamorphisms. Evidently, if $f$ is an equivalence, then $f$ is a Morita-morphism. Following [7] (also, see [8]), the cyclic (co)homology of $k$-category Morita equivalence. Using this fact and considering the deep results of [3], the following fact follows.

THEOREM 3.6. The reflexive cohomolgy and the dihedral (co)homology of the $k$ category $A$ with involution are invariant under Morita equivalence.

ACKNOWLEDGEMENT. This paper has been presented in the International Conference "Mathematics Today and Tomorrow" at the University of Central Florida, 13-15 March 1997.

\section{REFERENCES}

[1] B. L. Feĭgin and B. L. Tsygan, Additive $K$-theory, $K$-theory, Arithmetic and Geometry (Moscow, 1984-1986), Lecture Notes in Math., vol. 1289, Springer, Berlin, 1987, pp. 67-209. MR 89a:18017. Zbl 635.18008.

[2] A. Heller, Homological algebra in abelian categories, Ann. of Math. (2) 68 (1958), 484-525. MR 20\#7051. Zbl 084.26704.

[3] R. L. Krasauskas, S. V. Lapin, and Y. P. Solov'ev, Dihedral homology and cohomology. Basic notions and constructions, Math. USSR-Sb. 61 (1988), no. 1, 23-47, [translated from Mat. Sb. (N.S.) 133(175) (1987), no. 1, 25-48. MR 88i:18014. Zbl 654.18006.

[4] J.-L. Loday, Homologies diédrale et quaternionique [Dihedral and quaternionic homology], Adv. in Math. 66 (1987), no. 2, 119-148 (French). MR 89e:18024. Zbl 627.18006.

[5] _ Cyclic Homology, Grundlehren der Mathematischen Wissenschaften [Fundamental Principles of Mathematical Sciences], vol. 301, Springer-Verlag, Berlin, 1992. MR 94a:19004. Zbl 780.18009.

[6] J. M. Lodder, Dihedral homology and homotopy fixed point sets, Algebraic Topology (Oaxtepec, 1991) (Rhode Island), Contemporary Mathematics, vol. 146, Amer. Math. Soc., 1993, pp. 215-224. MR 94e:55014. Zbl 794.55006.

[7] S. I. Perelygin, Cyclic (co)homology of pre-additive categories, Dokl. Akad. Nauk BSSR 34 (1990), no. 11, 974-977 (Russian). MR 92d:19003. Zbl 716.18006. 
[8] C. A. Weibel, An Introduction to Homological Algebra, Cambridge Studies in Advanced Mathematics, vol. 38, Cambridge University Press, Cambridge, 1994. MR 95f:18001. Zbl 797.18001.

YASiEn Gh. Gouda: Department of MAthematics, FACUlty of SCience, SOUth VAlley UNIVERSITY, ASWAN, EGYPT

E-mail address: yasien10@hotmai 1. com 


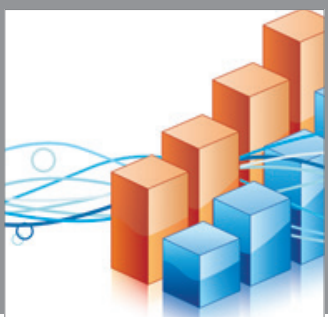

Advances in

Operations Research

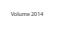

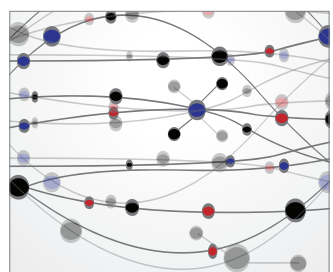

\section{The Scientific} World Journal
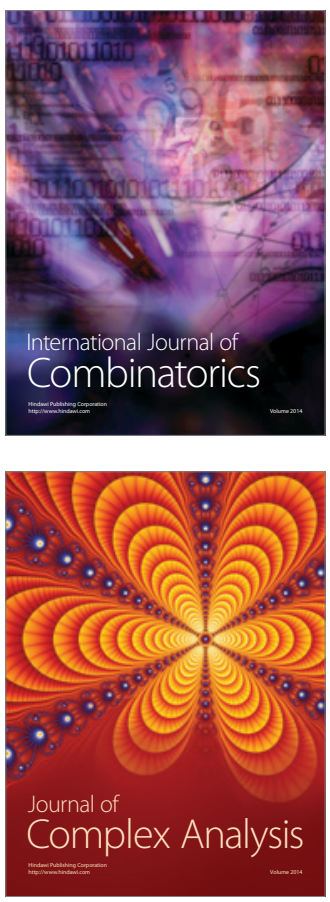

International Journal of

Mathematics and

Mathematical

Sciences
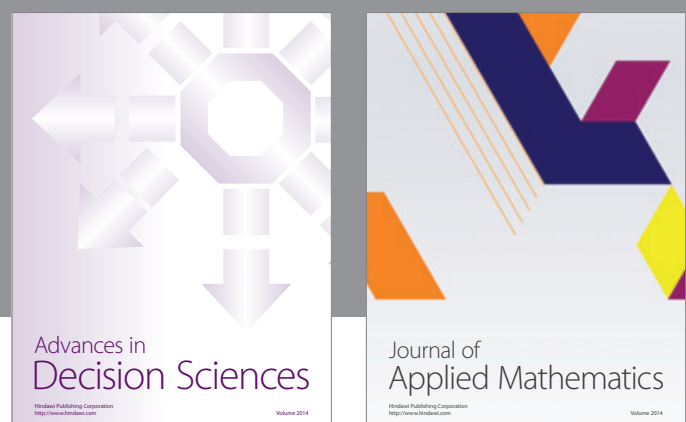

Journal of

Applied Mathematics
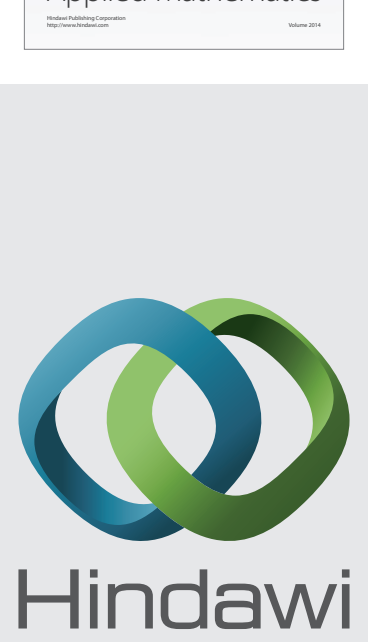

Submit your manuscripts at http://www.hindawi.com
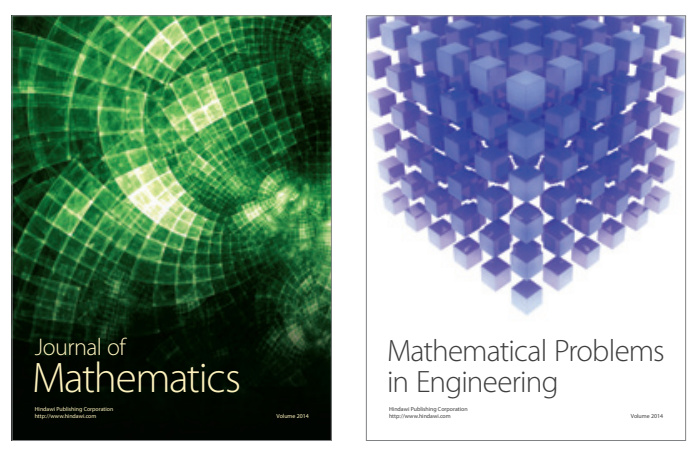

Mathematical Problems in Engineering
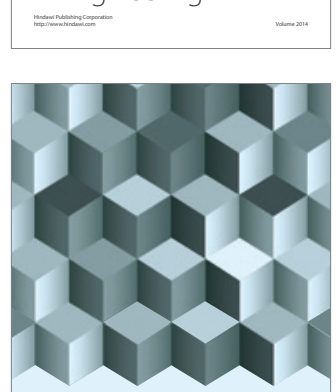

Journal of

Function Spaces
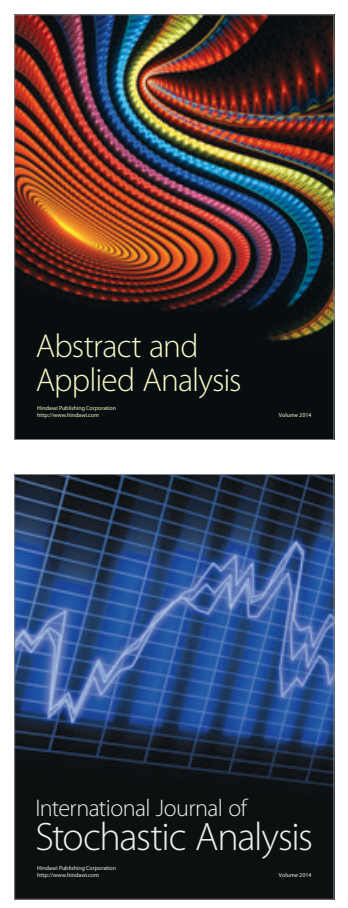

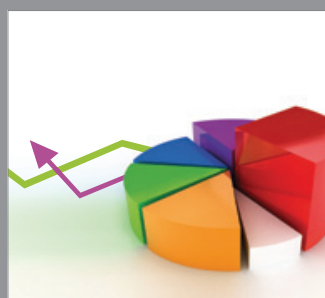

ournal of

Probability and Statistics

Promensencen
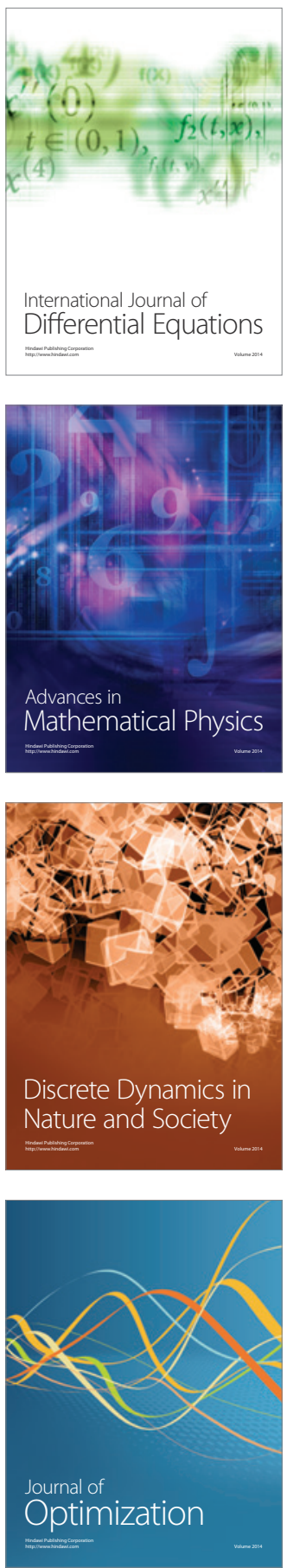